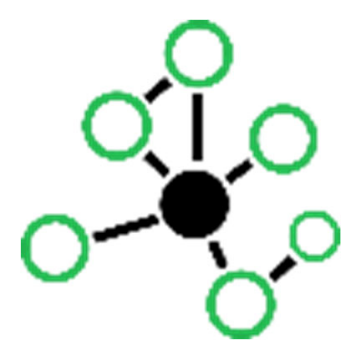

\title{
A flexible data architecture to automate collection of (near) real-time methane sensor data at commercial dairy farms
}

\author{
Kamphuis, C. ${ }^{1}$, Y. de Haas $^{1}$, E. van den Bergh ${ }^{2}$ \\ ${ }^{1}$ Wageningen Livestock Research, Animal Breeding and Genetics, De Elst 1, 6708 WD Wageningen, the Netherlands \\ ${ }^{2}$ Wageningen University and Research, Wageningen Data Competence Centre, Droevendaalsesteeg 2, 6708 PB \\ Wageningen, the Netherlands \\ Corresponding author's e-mail: claudia.kamphuis@wur.nl
}

To reduce methane emissions from the animal production domain, a large intensive research programme has been started in 2018 by the Dutch Ministry of Agriculture, Nature and Food. Part of this programme involves continuous monitoring of methane, by means of sensor measurements, both at the barn and individual animal level. This monitoring of methane will take place at several commercial dairy farms in the Netherlands. To collate the data from several methane sensors (so-called sniffers, but also lasers) in the field and to link this information to individual barns and animals, a number of challenges have to be tackled: sensor functioning and data quality should be monitored continuously to bring loss of data to a minimum, and data transfer from the sensor to a storage platform should be cheap and trustworthy. Moreover, the platform should be flexible in accepting other types of information (e.g., other sensor data, but also animal and farm data that are often stored in separate farm management programs) and in handling data from new farms in case the number of farms with methane measurements will increase. The objective of this project is to automate and unify current data flows into a single platform. Sensor output will be gathered and sent using low-power, small form factor devices. To ensure easy placement on any farm, independent of local internet connectivity, a long-range radio protocol will be used to send the data to the cloud. Data will be gathered using the IoT-hub presented by Microsoft Azure which will provide basic monitoring and alerts for sensor performance. Gathered data will be exposed through Application Programming Interfaces (APIs) to enable monitoring apps for a range of end-users. To enable exploration of the data, the data will be mirrored to an ElasticSearch instance. Together, these techniques will enable a safe, flexible and scalable data platform that will enable scientists, policy makers and farmers to use these data whenever needed. This project now uses methane emission at a small number of dairy farms as use case. However, in case this proof of principle works, it could be installed in many more dairy barns, but also in barns with other species (pigs, poultry, goats, calves) when recording greenhouse gas emissions. 